\title{
Indocyanine green-loaded injectable alginate hydrogel as a marker for precision cancer surgery
}

\author{
Seon Sook Lee ${ }^{1}$, Hyunjin Kim ${ }^{1}$, Dae Kyung Sohn², Joo Beom Eom ${ }^{3}$, Young Seok Seo ${ }^{4}$, Hong Man Yoon ${ }^{5}$, \\ Yongdoo Choi ${ }^{1}$
}

${ }^{1}$ Research Institute, ${ }^{2}$ Center for Colorectal Cancer, National Cancer Center, Goyang, Republic of Korea; ${ }^{3}$ College of Medicine, Dankook University, Cheonan, Republic of Korea; ${ }^{4}$ R\&D Center, Wontech Co., Ltd., Daejeon, Republic of Korea; ${ }^{5}$ Center for Gastric Cancer, National Cancer Center, Goyang, Republic of Korea

Correspondence to: Yongdoo Choi, PhD. Research Institute, National Cancer Center, 323 Ilsan-ro, Goyang, Gyeonggi 10408, Republic of Korea. Email: ydchoi@ncc.re.kr.

Background: Accurate identification of tumor sites and boundaries is of paramount importance during minimally invasive surgery. Although laparoscopic resection is being increasingly and widely performed for early gastric and colorectal cancers, the detection of tumors located inside the stomach and intestine is difficult owing to the lack of tactile sensation. Here, we propose the application of an indocyanine green (ICG)-loaded alginate hydrogel system as a fluorescence surgical marker for precise laparoscopic operations. Methods: A physical complex of ICG and human serum albumin (HSA) was mixed with sodium alginate to form an injectable hydrogel system. Calcium carbonate and D-gluconic acid (GA) were added to the gel to control its strength and gelation time, respectively. The optimal conditions for the preparation of injectable hydrogels were determined by analyzing the fluorescence spectra and sol-gel transition time of the prepared samples at various concentrations and compositions. Next, the aqueous solutions of ICG, ICG-HSA, and ICG-HSA-loaded alginate were subcutaneously injected into nude mice (three mice per group), and nearinfrared (NIR) fluorescence images of the mice $\left(\lambda_{\text {ex. }}=780 \mathrm{~nm}, \lambda_{\text {em. }}=845 \mathrm{~nm}\right)$ were obtained at different points in time for 8 days. Then, fluorescence intensities at the injection sites, target-to-background ratio, and areas of ICG fluorescence were analyzed. Finally, the potential utility of ICG-HSA-loaded alginate hydrogel as a surgical marker was evaluated in a porcine model. The ICG-HSA-loaded alginate solution was injected into three sites in the submucosal space of the porcine stomach via a catheter. A fluorescent laparoscopic system was installed on the abdomen of the pig 3 days post-injection, and the fluorescence signal generated from the fluorescence surgical marker located inside the stomach was evaluated using the fluorescence laparoscope $\operatorname{system}\left(\lambda_{\text {ex. }}=785 \mathrm{~nm}, \lambda_{\text {em. }}=805 \mathrm{~nm}\right)$.

Results: The optimal concentration of ICG-HSA complex was determined to be $30 \mu M$, and maximum fluorescence intensity of the complex was obtained at a 1:1 mole ratio of HSA to ICG. The subcutaneous injection of ICG or ICG-HSA solution in mice resulted in the rapid spread of the fluorescence signal around the injection site in $3 \mathrm{~h}$, and a weak fluorescence was detected at the injection site $24 \mathrm{~h}$ post-injection. In contrast, the fluorescence detection time was effectively prolonged up to $96 \mathrm{~h}$ post-injection in the case of ICG-HSA-loaded alginate gel, while diffusion of the injected ICG from the injection site was effectively prevented. In the laparoscopic operation, injection sites of the hydrogel in porcine stomach could be accurately detected in real time even after 3 days.

Conclusions: This alginate hydrogel system may be potentially useful as an effective surgical marker in terms of accuracy and persistence for laparoscopic operation.

Keywords: Alginic acid; hydrogel; indocyanine green (ICG); laparoscopy; surgical marker

Submitted Jan 03, 2020. Accepted for publication Feb 26, 2020.

doi: 10.21037/qims.2020.02.24

View this article at: http://dx.doi.org/10.21037/qims.2020.02.24 


\section{Introduction}

Laparoscopic surgery, a minimally invasive procedure, reduces the impact on the patient's body and allows surgeons to perform operations through small holes (usually $0.5-1.5 \mathrm{~cm}$ long) as compared to traditional operations (usually through 24-36 cm long holes) (1). Past several decades have noticed development in laparoscopic surgery owing to advantages such as less bleeding and pain, rapid recovery rate, and shortened hospitalization period as compared with traditional open surgery (2). However, palpation of suspicious regions for tumor detection is impossible with laparoscopic operation unlike conventional surgery, and it is difficult to detect small soft lesions or polypectomy sites (3). Therefore, the incision range may vary according to the skills of the operator. To reduce this error and improve the accuracy of operation, it is imperative to preoperatively mark the surgical sites. To improve tumor detection, several marking methods have been developed, including fluoroscopy location (4), barium imaging (5), marking with metal clips $(6,7)$, and tattooing using dyes such as methylene blue or India ink (8). However, each of these methods suffer from limitations. Intraoperative fluoroscopy is expensive and time-consuming, may need bowl distension, and increases contamination rate (9). Barium imaging demand the use of radiation (5) and may be unreliable (10). Metal clips are expensive and invisible outside, and may dislodge before or during surgical operation $(5,8)$. Direct injection of a dye into the bowel may result in its diffusion and spilling into the peritoneal cavity and cause fat necrosis, focal perforation, or peritonitis $(11,12)$. Therefore, development of a new surgical marker and an imaging method is desirable.

Fluorescence dyes with absorption and emission peaks in the near-infrared (NIR) wavelength range (650-900 nm) have been considered to serve as excellent agents to mark tumor sites (13). NIR fluorescence imaging allows detection of fluorescence signals in deep tissues by minimizing autofluorescence in the NIR wavelength range $(14,15)$. Moreover, fluorescence signals may be sensitively detected using only a small amount of dye (nanomolar concentrations) in vivo (16). Indocyanine green (ICG), an FDA-approved NIR dye, is widely used as an imaging agent in medical diagnosis. However, its applications as a surgical marker are limited owing to the low fluorescence intensity resulting from the concentration-dependent coagulation, instability in aqueous solutions, and rapid diffusion at the site of injection (17).
Herein, we propose an injectable alginate hydrogel containing an ICG-human serum albumin (HSA) complex as a new surgical marker for minimally invasive surgery (Figure 1). Alginate is a polysaccharide resembling glycosaminoglycan as an extracellular matrix. It is a natural polymer extracted from brown algae; the excellent biocompatibility and hydrophilic characteristic have extended the use of alginate for biomedical applications, including soft tissue engineering and biomolecular delivery (18). As alginate is a polyanionic polymer (19), it can easily form a gel through ionic cross linking with divalent ions (e.g., $\left.\mathrm{Ca}^{2+}\right)(18,20)$, while it is not cleaved in mammals owing to the lack of suitable enzymes $(21,22)$. In addition, the gelling time may be modulated by adjusting concentrations and types of ions and additives added to alginate solution (19).

We expected the following improvements through this system. First, as ICG exhibits high affinity for proteins (23), it may easily bind to HSA and show higher fluorescence efficiency and stability than free ICG. Second, as a scaffold for the ICG-HSA complex, alginate hydrogel would prevent the rapid diffusion of the injected dye and prolong the fluorescence detection time.

\section{Methods}

\section{Materials}

Sodium alginate was obtained from KIMICA Incorporation (Tokyo, Japan, viscosity 500-600 mPass). Calcium carbonate $\left(\mathrm{CaCO}_{3}\right)$, D-gluconic acid (GA) solution, HSA, and ICG were purchased from Sigma-Aldrich (St. Louis, MO, USA). Dialysis membrane (MW cut-off: $50 \mathrm{kDa}$ ) was supplied by Spectrum Laboratories (Laguna Hills, CA, USA).

\section{Determination of optimal conditions for ICG-HSA complex formation}

The optimal concentration of ICG-HSA complex was determined. ICG $(1.5 \mathrm{mg})$ was dissolved in $3 \mathrm{~mL}$ deionized (DI) water to obtain a concentrated ICG solution. To prepare ICG-HSA complex, $1 \mathrm{~mL}$ of concentrated ICG solution was added to $42.89 \mathrm{mg}$ HSA at a 1:1 (ICG:HSA) molar ratio. The concentrated ICG-HSA solution was stirred at $25{ }^{\circ} \mathrm{C}$ for $1 \mathrm{~h}$ for stabilization. The ICG-HSA solution was then diluted with DI water to $2-100 \mu \mathrm{M}$ range. Fluorescence spectra of ICG-HSA solutions $\left(\lambda_{\text {ex }}=720 \mathrm{~nm}\right.$, $\left.\lambda_{\text {em. }}=740-850\right)$ were measured with a multifunctional 


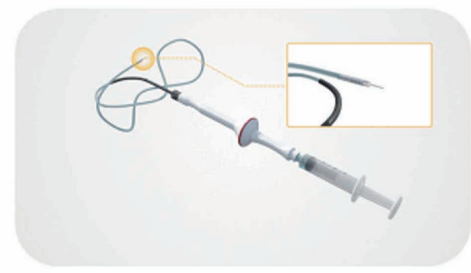

Injectable Hydrogel System

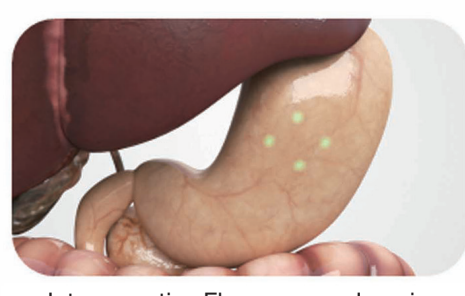

Intraoperative Fluorescence Imaging

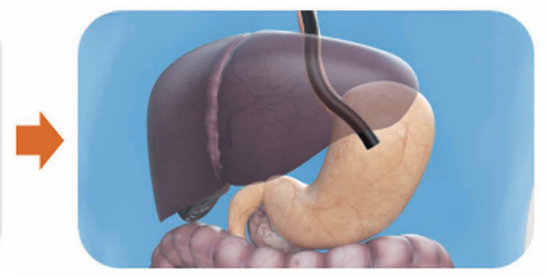

Endoscopy

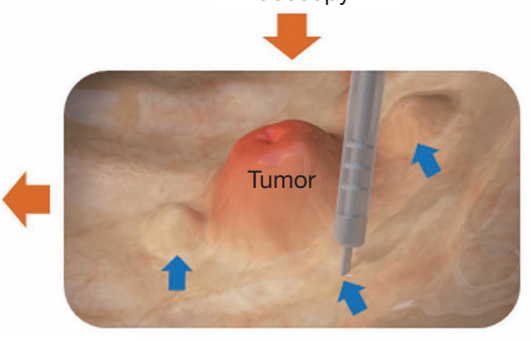

Injection and Hydrogel Formation

Figure 1 Schematic illustration of marking surgical sites with an ICG-HSA-loaded alginate hydrogel and subsequent fluorescence detection of the marking sites in real-time.

Table 1 The compositions of alginate hydrogels in a dual syringe

\begin{tabular}{lccc}
\hline Sample & $\# 1$ & $\# 2$ & $\# 3$ \\
\hline Solution A & 1.0 & 1.0 & 1.0 \\
$\quad$ Alginate (wt/v\%) & $0.2 \times$ & $0.4 \times$ & $0.6 \times$ \\
$\mathrm{CaCO}_{3}$ & & & \\
Solution B & 1.0 & 1.0 & 1.0 \\
Alginate (wt/v\%) & $0.4 \times$ & $0.8 \times$ & $1.2 \times$ \\
D-Gluconic acid & & & \\
\hline
\end{tabular}

$\times$, Number of moles of carboxylic acid in $1.0 \mathrm{wt} / \mathrm{v} \%$ alginate solution.

microplate reader (Safire 2, Tecan, Männedorf, Switzerland).

The optimal ratio of ICG versus HSA to obtain maximum fluorescence intensity was evaluated. ICG concentration in DI water was fixed to $30 \mu \mathrm{M}$, and ICG was mixed with various molar ratios of HSA. The fluorescence spectra of the solution $\left(\lambda_{\text {ex. }}=720 \mathrm{~nm}, \lambda_{\text {em. }}=740-850\right)$ were obtained and compared.

\section{Analysis of gelation time for alginate hydrogels containing $\mathrm{CaCO}_{3}-\mathrm{GA}$}

Sodium alginate was dissolved in DI water, dialyzed for 2 days ( $\mathrm{MWCO}=50 \mathrm{kDa}$ ), and then freeze dried.

Sodium alginate in DI was mixed with different amounts of $\mathrm{CaCO}_{3}$ to obtain solution A (see Table 1 for the concentration of sodium alginate and $\left.\mathrm{CaCO}_{3}\right)$. Solution $\mathrm{B}$ was prepared by mixing sodium alginate solution with different concentrations of GA solution. The molar ratio of GA to $\mathrm{CaCO}_{3}$ was adjusted to 2 for the neutralization of the $\mathrm{pH}$ of the final solution (22). Solutions $\mathrm{A}(0.35 \mathrm{~mL})$ and $\mathrm{B}$ $(0.35 \mathrm{~mL})$ were separately charged into a dual syringe and simultaneously injected into a $5 \mathrm{~mL}$ glass vial to prepare an in situ gel. Sol-gel transition time of alginate/ $\mathrm{Ca}^{2+}$ GA composite solutions was determined by the test-tube inverting method (24) by inverting the tube every $1 \mathrm{~min}$ after injection. The solution state was determined by flow (sol) and non-flow state (gel).

\section{In vivo evaluation of alginate hydrogels in a mouse model}

The potential utility of ICG-HSA-loaded alginate hydrogel 
as a surgical marker was evaluated in a mouse model. ICG, ICG-HSA solution, and ICG-HSA-loaded alginate/ $\mathrm{Ca}^{2+}$-GA composite system (alginate hydrogel) were subcutaneously injected into male nude mice (6 weeks, $\mathrm{n}=3$ per group). The final concentrations of sodium alginate, ICG-HSA, $\mathrm{CaCO}_{3}$, and GA in the injected gel were $1 \mathrm{wt} / \mathrm{v} \%, 30 \mu \mathrm{M}, 0.3 \times$, and $0.6 \times$, respectively. The injection volume of the sample solution was $100 \mu \mathrm{L}$. NIR fluorescence images $\left(\lambda_{\text {ex. }}=780 \mathrm{~nm}, \lambda_{\text {em. }}=845 \mathrm{~nm}\right)$ of mice were obtained using the IVIS Lumina XR system (Xenogen Corp., Alameda, CA, USA) at different time points for 8 days. The animal experiment protocol was approved by the Institutional Animal Care and Use Committee (IACUC) of the National Cancer Center (approval No. NCC-18451) of Korea.

For quantitative analysis of fluorescence intensities in the region of interest (ROI), target-to-background ratio (TBR) and area of ICG fluorescence were determined using an imaging software. TBR was determined as follows: TBR = ROI (target)/ROI (background) (25).

\section{In vivo evaluation of alginate hydrogels in a porcine model}

A healthy male landrace pig $(45 \mathrm{~kg}$ ) was obtained from Cronex Co., Ltd. (Hwaseong, Korea). All experiments were conducted in KNOTUS Co., Ltd (Incheon, Korea) and approved by the IACUC of KNOTUS (approval No. 19-KE-358).

The pig was fed with a semifluid diet for $48 \mathrm{~h}$ prior to experiment inception and fed with a polyethylene glycol solution on the day prior to endoscopy. General anesthesia was administered with an injection of Zoletil 50 (VIRBAC, France, $5 \mathrm{mg} / \mathrm{kg}$ ) and xylazine (Rompun ${ }^{\circledR}$, Bayer AG, Germany, $2.5 \mathrm{mg} / \mathrm{kg}$ ). The pig was intubated and anesthesia was maintained with $1-2 \%$ isoflurane added to the oxygen gas by a ventilator. The pig was administered a 50-cc saline enema through the anus until all residual fecal material was evacuated. An endoscope with an attached cap was advanced into the stomach of the pig.

ICG-HSA-loaded alginate solution was then injected into three sites ( $1 \mathrm{~mL}$ solution per site) of the submucosal space in the porcine stomach via catheter $(23 \mathrm{G}$ needle Clear-Jet injector, Finemedix, Daegu, Korea). To mark injection sites, hemostatic clips were clipped right beside the injection sites. After 3 days of hydrogel injection, a fluorescent laparoscopic system was installed on the abdomen of the pig, and the fluorescence signal generated from the fluorescence surgical marker located inside the stomach was evaluated in real time using a fluorescence laparoscope system. The pig was euthanized, and the stomach was dissected to verify that the position identified as the gel injection site by the fluorescence image was accurate (26).

\section{NIR fluorescence laparoscopic system}

Fluorescence light source is composed of white and infrared light (Figure 2). White light has a color temperature of $5,500 \mathrm{~K}$ at a maximum output of 2,600 $\mathrm{lm}$ and an infrared wavelength of $785 \mathrm{~nm}$ at a maximum output of $3.5 \mathrm{~W}$. Two light sources are mixed with a $5 \mathrm{~mm}$ diameter fiber bundle and irradiated onto the image target using a laparoscope $\left(\lambda_{\text {ex. }}=785 \mathrm{~nm}, \lambda_{\text {em. }}=805 \mathrm{~nm}\right)$. Laparoscopes are designed to target images $50 \mathrm{~mm}$ apart with a $10 \mathrm{~mm}$ diameter and $70^{\circ}$ field of view.

The eyepiece of the laparoscope was attached to a camera head to simultaneously acquire color and fluorescence images. The camera head comprised a coupler lens and an image-accepting sensor. The coupler lens clarifies the focus of the image targeted by the laparoscope and delivers the image to the sensor. The image-accepting sensor passes the data to an imaging system for image construction.

\section{Results}

\section{Optimal condition for ICG-HSA complex formation}

To determine the optimal concentration of ICG-HSA, fluorescence spectra were obtained after mixing ICG and HSA at different concentrations and mole ratios. The ICGHSA complex (1:1 mole ratio) showed a continuous increase in the fluorescence intensity in the concentration range of 2-30 $\mathrm{MM}$ (Figure 3A,B). The maximum fluorescence intensity remained unchanged above $30 \mu \mathrm{M}$. Further, the change in the fluorescence intensity of ICG-HSA complex was analyzed by varying the mole ratio of HSA to ICG (from 0 to 2; Figure 3C,D). As a result, the fluorescence intensity of the complex solution increased as the amount of HSA in the ICG-HSA complex increased. In particular, the fluorescence signal increased until the two substances were combined at a one-to-one ratio, while only a little change was observed with any further increase in HSA. Therefore, we selected the one-to-one combination of ICG-HSA in subsequent experiments.

\section{Gelation time of alginate hydrogels containing $\mathrm{CaCO}_{3}-\mathrm{GA}$}

To prepare alginate hydrogel with an adequate gelation time 


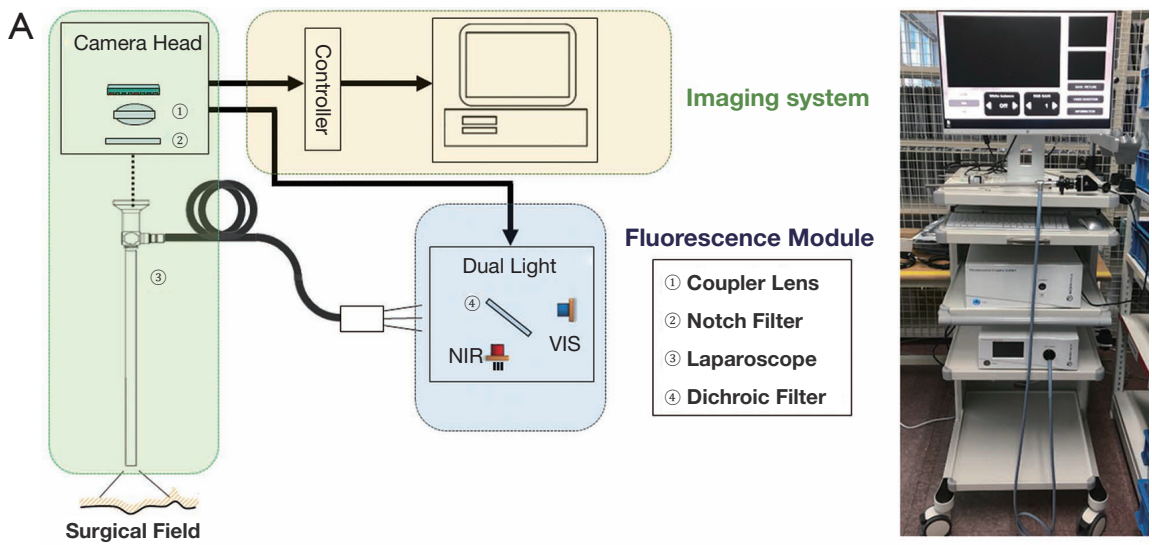

B

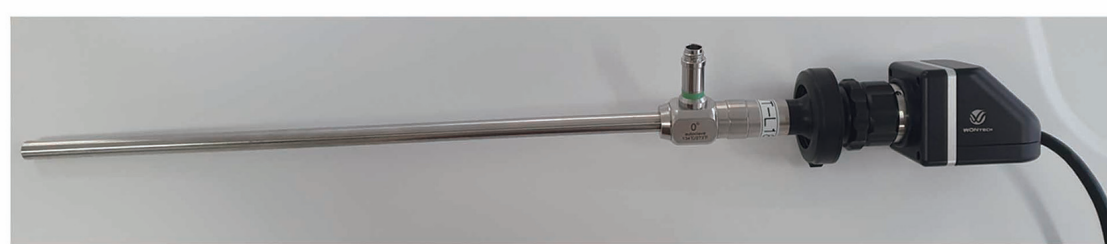

Figure 2 Real-time NIR fluorescence laparoscopic system. (A) Schematic diagram and the assembled system for laparoscopic fluorescence imaging; (B) the constructed laparoscope with coupling lens and camera head.
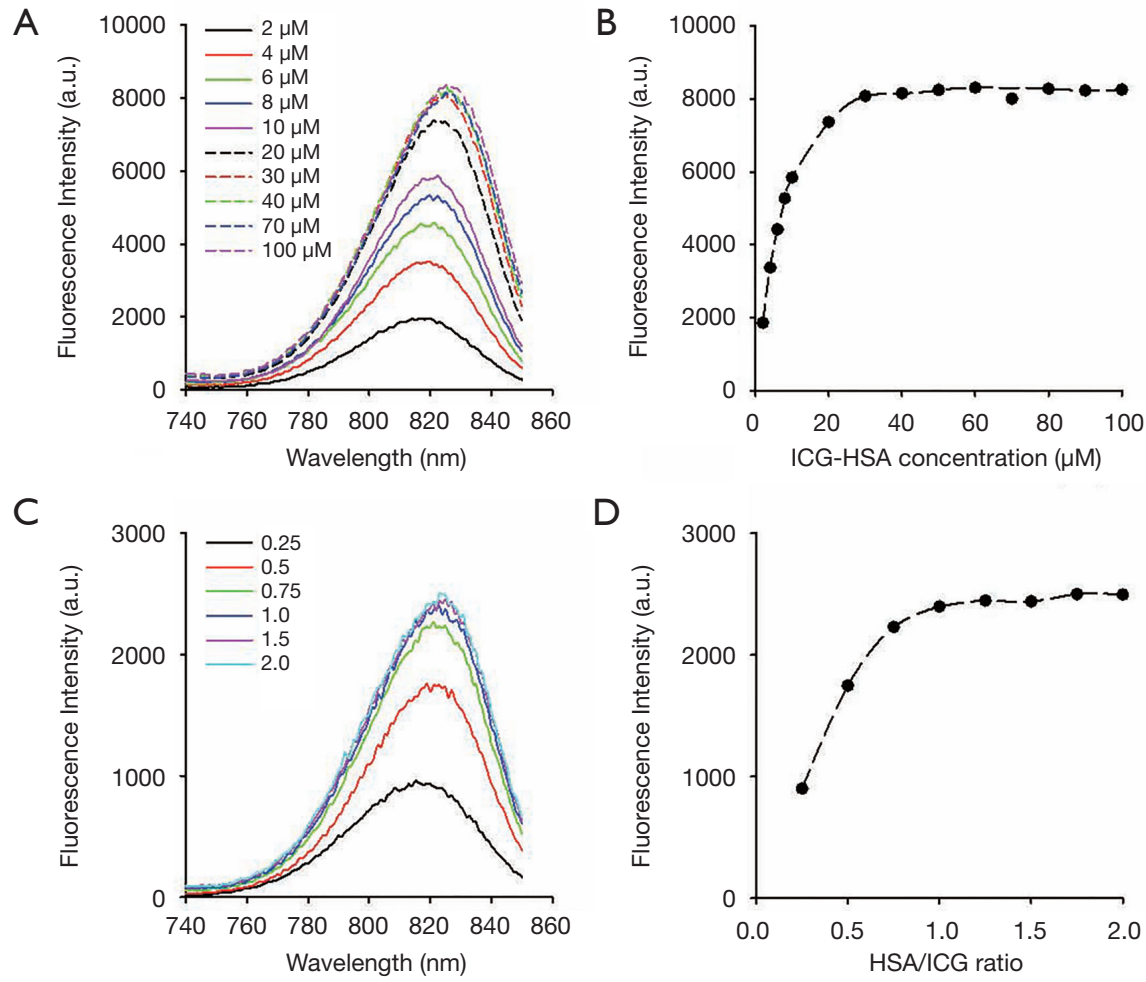

Figure 3 In vitro fluorescence data. (A) Fluorescence spectra of ICG-HSA complexes $\left(\lambda_{\text {ex. }}=720\right.$ nm) at various concentrations; (B) peak fluorescence intensity (molar ratio of ICG:HSA $=1: 1 ; \lambda_{\text {ex. }}=720 \mathrm{~nm}, \lambda_{\text {em. }}=823 \mathrm{~nm}$ ) versus ICG-HSA concentration; $(C)$ fluorescence spectra $\left(\lambda_{\text {ex. }}\right.$ $=720 \mathrm{~nm}$ ) of ICG-HSA complexes at different HSA/ICG ratios; (D) peak fluorescence intensity $\left(\mathrm{ICG}=30 \mu M ; \lambda_{\text {ex. }}=720 \mathrm{~nm}, \lambda_{\text {em. }}=823 \mathrm{~nm}\right)$ versus HSA/ICG molar ratio. ICG, indocyanine green; HSA, human serum albumin. 
A

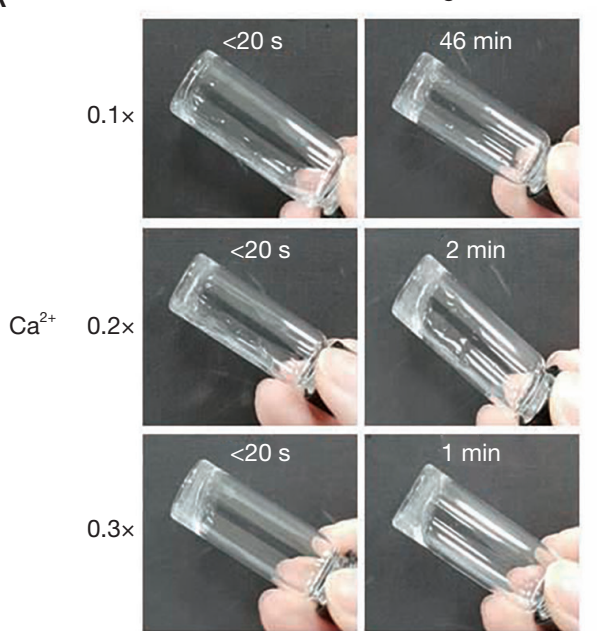

B

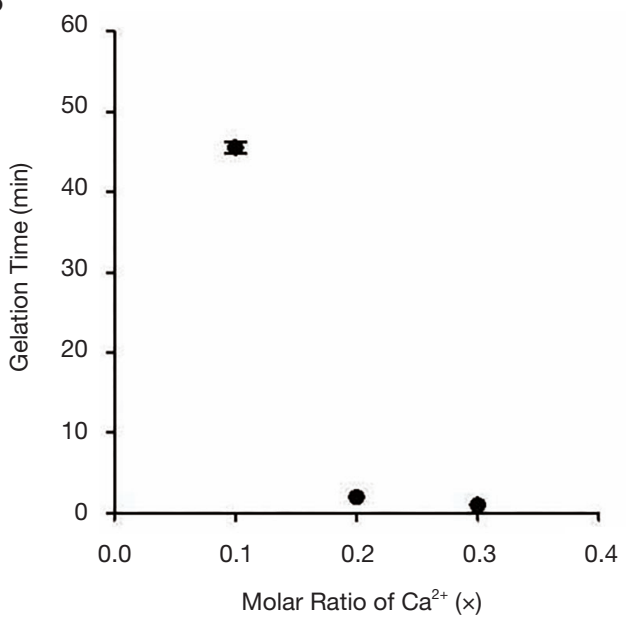

Figure 4 Effect of calcium ions on gelation time. (A) Images of gels formed at different time points after injection with a dual syringe; (B) gelation time of alginate hydrogels as a function of $\mathrm{Ca}^{2+}$ concentration $(x$ indicates the mole number of carboxylic acids in $1.0 \mathrm{wt} / \mathrm{v} \%$ alginate solution).

as a surgical marker, different concentrations of calcium ions were mixed with the alginate solution using a dual syringe (Table 1). Gelation time of the alginate solution was evaluated.

As shown in Figure $4 A$, the alginate solution containing $0.1 \times$ calcium ion remained in its liquid state (sol) for about $46 \mathrm{~min}$ after injection before forming a solid gel. Under $0.2 \times$ calcium concentration, the solution was in a slightly liquid state (sol) immediately after injection and changed to the solid gel state after about $2 \mathrm{~min}$. The solution exposed to $0.3 \times$ calcium concentration formed a weak gel immediately after injection and changed to a solid gel state after about $1 \mathrm{~min}$. The gelation time is shown in Figure $4 B$ according to the final concentration of calcium ions in the injected gel. The gelation time of alginate was shortened as the concentration of calcium ion increased from $0.1 \times$ to $0.3 \times$. In particular, the alginate solution treated with $0.3 \times$ calcium existed in a solid state (gel) immediately after injection. Therefore, $0.3 \times$ calcium concentration was selected for further experiments.

\section{In vivo evaluation of alginate hydrogels in a mouse model}

We next evaluated the ICG-HSA-loaded alginate hydrogel as a surgical marker in vivo. The aqueous solutions of ICG, ICG-HSA, ICG-HSA-loaded alginate were subcutaneously injected into nude mice using a dual syringe (Figure $5 A$ ). Then, NIR fluorescence images and photographs of the mice were periodically obtained (Figure $5 B, C)$. As a result, the fluorescence intensities at ICG and ICG-HSA injection sites rapidly decreased with time, and most of the fluorescence signals disappeared after $24 \mathrm{~h}$ from injection. Quantitative analyses of the fluorescence intensity and area of fluorescence emission with time (Figure 6) showed that most of the injected ICG and ICGHSA complex rapidly diffused into the surrounding tissues and disappeared from the injection sites within $24 \mathrm{~h}$. Only $16 \%$ and $12 \%$ fluorescence signals were retained at ICG and ICG-HSA injection sites, respectively, relative to the signal detected at the start. In contrast, the mice receiving alginate hydrogel retained $61 \%$ fluorescence signal 48 $\mathrm{h}$ post-injection, while significant NIR fluorescence persisted at the injection sites for about 4 days. The area of fluorescence emission was maintained at low values for several days, indicating that the alginate hydrogel effectively prevented the diffusion of the ICG-HSA complex from the injection site.

Figure $5 C$ shows the images of the injection site of ICG, ICG-HSA solution, and alginate hydrogel in nude mice. In particular, the injection sites for ICG and ICGHSA solutions appeared slightly swollen immediately after injection, and the solutions spread and became invisible after about $24 \mathrm{~h}$. On the other hand, protrusions formed immediately after injection in the alginate hydrogel-treated mice, and were retained for 5 days. 

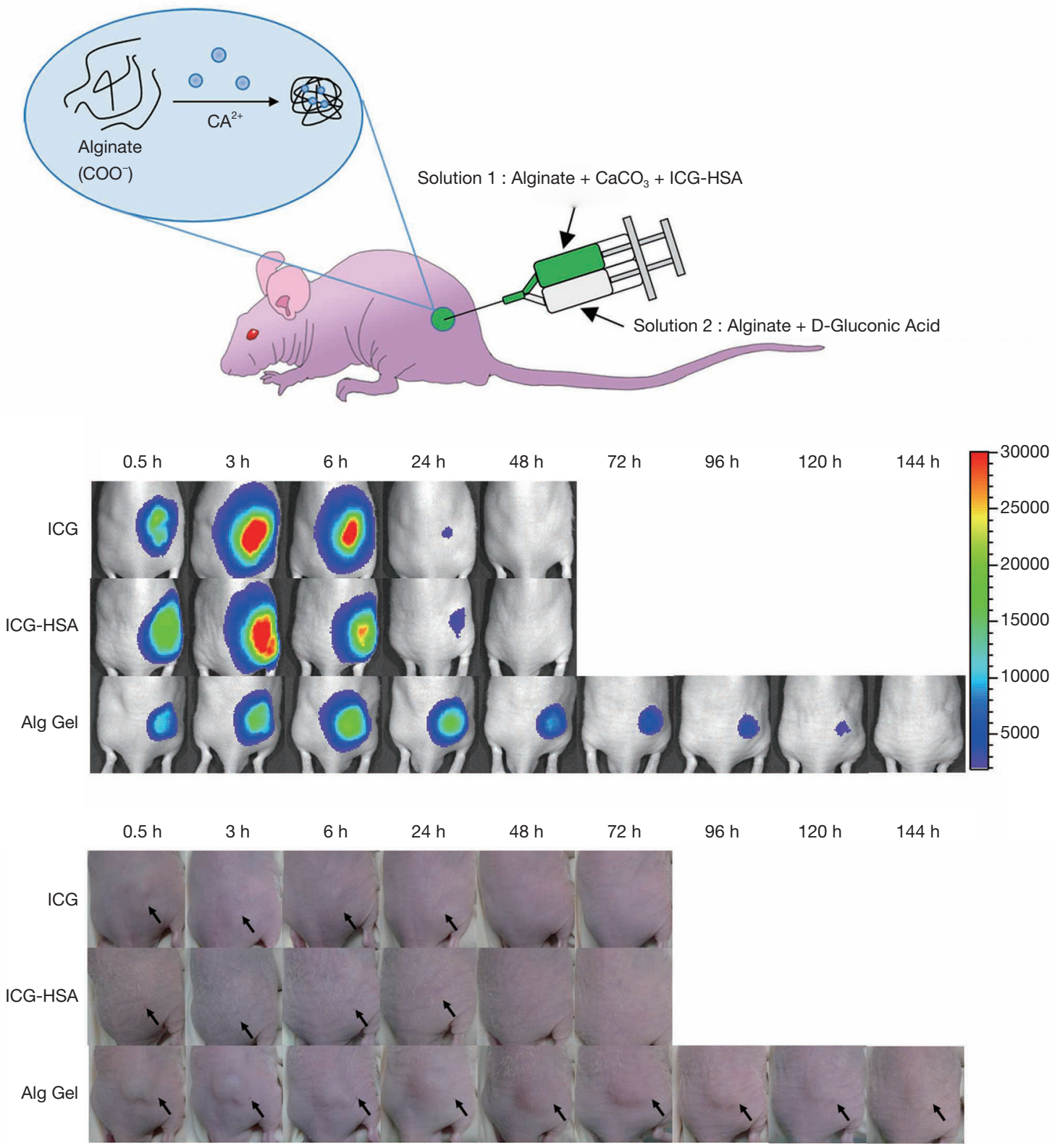

Figure 5 In vivo evaluation of ICG-HSA alginate hydrogel in a mouse model. (A) Scheme of the subcutaneous injection of ICG-HSAloaded alginate solution into a nude mouse using a dual syringe; (B) NIR fluorescence images of mice obtained using the IVIS Lumina XR system $\left(\lambda_{\text {ex. }}=780 \mathrm{~nm}, \lambda_{\text {em. }}=845 \mathrm{~nm}\right)$ and $(\mathrm{C})$ images of injection sites in nude mice at defined time intervals. Arrows indicate the injection sites of ICG, ICG-HSA, and ICG-HSA-loaded alginate hydrogel (Alg Gel) in mice. ICG, indocyanine green; HSA, human serum albumin; NIR, near-infrared.

\section{In vivo evaluation of alginate hydrogels in a porcine model}

The potential application of ICG-HSA-loaded alginate hydrogel as a surgical marker was evaluated in a porcine model. The solution was injected into the submucosal space of the porcine stomach using an injector. Hemostatic clips were clipped right beside the injection sites. After 3 days from injection, NIR fluorescence imaging was performed for the injected sites using a fluorescence laparoscopic system (Figure $7 A$ ). As a result, the location and margins 

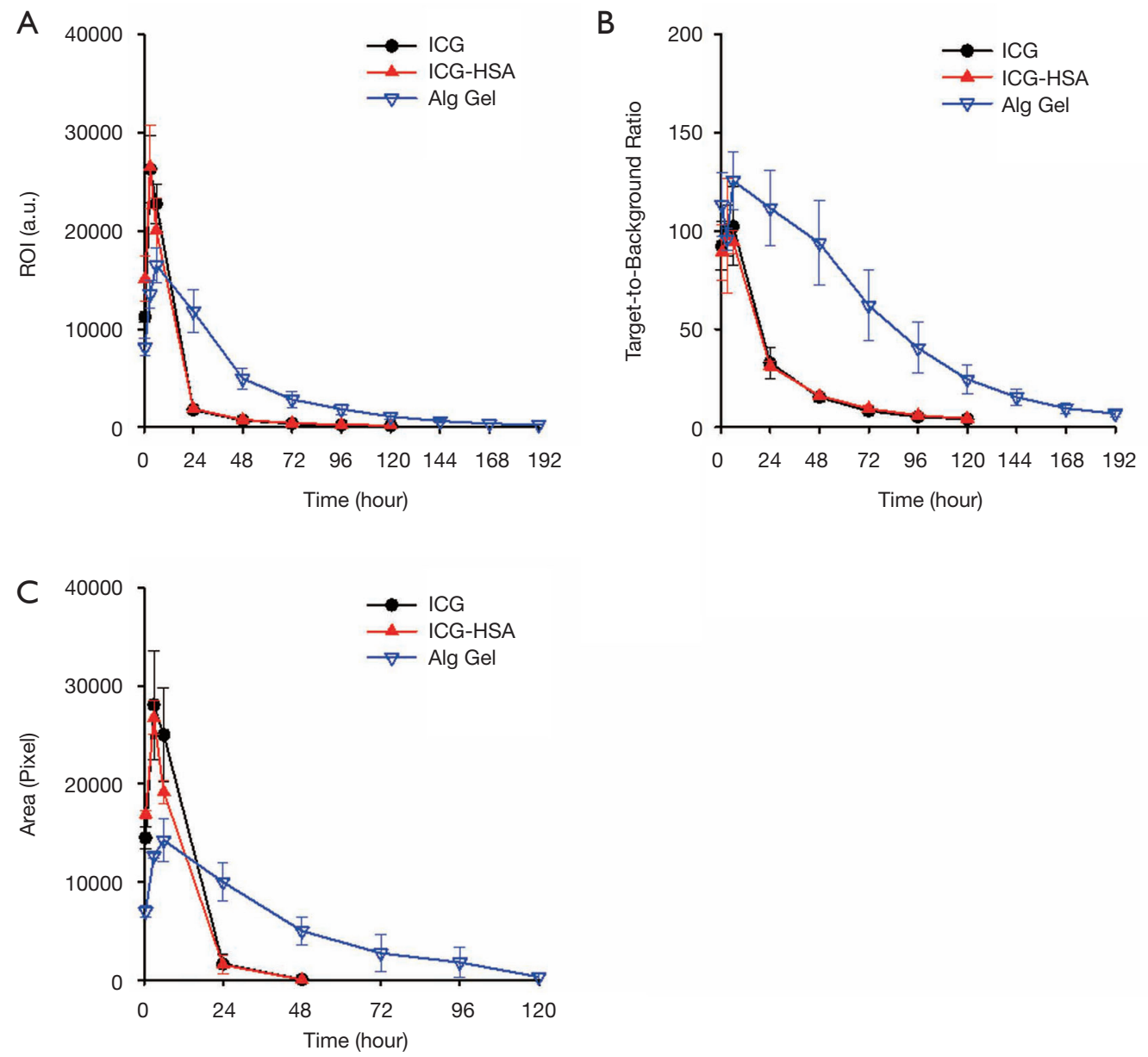

Figure 6 Quantitative analyses of NIR fluorescence images. (A) Region of interest, (B) target-to-background ratio, and (C) area of fluorescence emission in nude mice at defined time intervals. NIR, near-infrared.

of injection sites could be clearly distinguished in real time using NIR fluorescence images (Figure 7B).

To ensure that the fluorescence signal was emitted from the injected hydrogel, porcine stomach was opened and the fluorescence was evaluated ex vivo (Figure 7C). Strong fluorescence signal was detected right beside the hemostatic clip, confirming that the NIR fluorescence signal detected using fluorescence laparoscopic system was derived from the injection sites of ICG-HSA-loaded alginate hydrogels.

\section{Discussion}

The incidence of early gastrointestinal cancers has been increasing, resulting in the increase in popularity of the use of the laparoscopic system for related surgeries. However, real-time detection of small lesions is difficult due to the lack of tactile sensation using the laparoscopic system. Therefore, ICG-HSA-loaded alginate hydrogels were developed as a novel surgical marker for the non-invasive and real-time detection of cancer sites, enabling precision cancer surgery.

As depicted in Figure 5B, the ICG-HSA complex initially emits strong fluorescence signals at the injection site; however, the signals rapidly decrease within $24 \mathrm{~h}$ due to dye diffusion from the injection site to the surrounding tissues. Alginate was mixed with both ICG-HSA and an ion crosslinker (e.g., $\mathrm{Ca}^{2+}$ ) to form a hydrogel, successfully inhibiting the diffusion of the ICG dyes from the injection site. Because the solution is injected through a catheter, it is essential to form a gel after injection to prevent jamming. Calcium chloride $\left(\mathrm{CaCl}_{2}\right)$ has been widely used for the preparation of hydrogels; however, gel formation after 

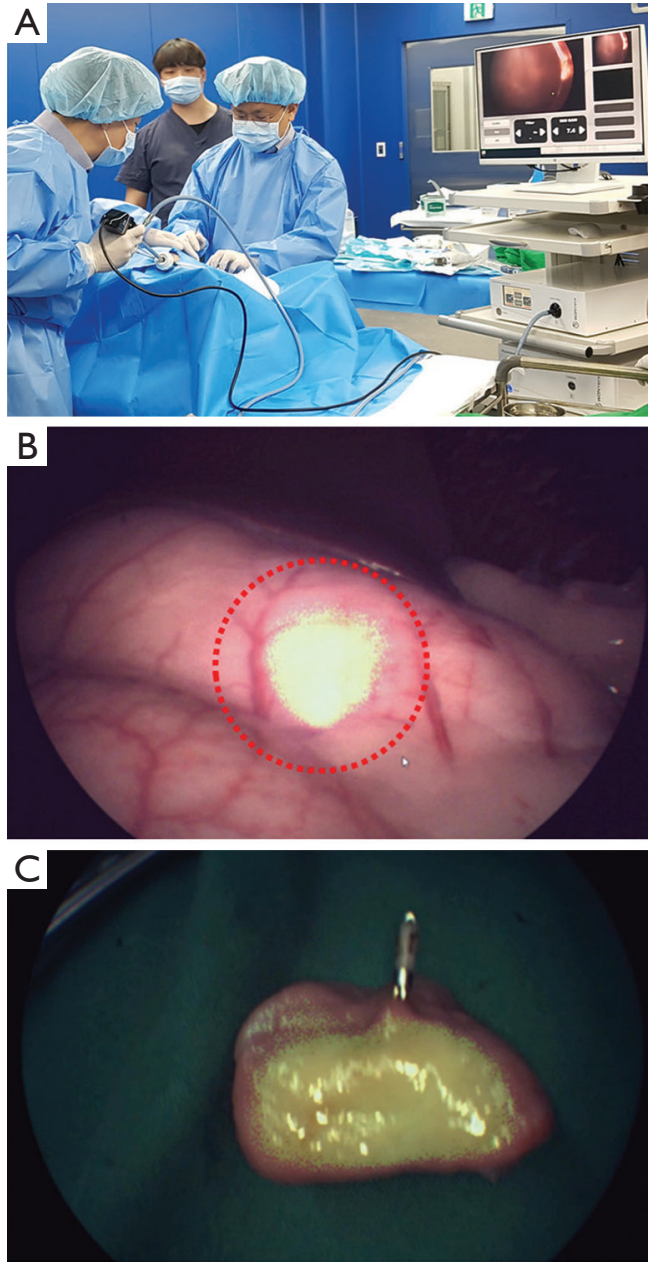

Figure 7 In vivo evaluation of ICG-HSA-loaded alginate hydrogels in a porcine model. (A) Image of the intraoperative identification of the injection site using laparoscopic fluorescence imaging system; (B) in vivo NIR fluorescence image obtained with fluorescence laparoscopy; (C) ex vivo NIR fluorescence image of the stomach tissue 3 days after injection. Clip is located right beside the injection site. ICG, indocyanine green; HSA, human serum albumin; NIR, near-infrared.

$\mathrm{CaCl}_{2}$ addition is excessively rapid. It is also difficult to form a uniform gel and the injector line gets easily clogged. Therefore, calcium carbonate $\left(\mathrm{CaCO}_{3}\right)$ was used in this study as a source of calcium ions to delay the gelation time (21). GA in solution B was added to facilitate $\mathrm{CaCO}_{3}$ dissolution at the injection site.

In vivo animal studies in mouse and porcine models confirmed that dye diffusion from the injection sites could be effectively prevented using the ICG-HSA-loaded alginate hydrogel. Thus, strong fluorescence at the injection sites was maintained for more than 3 days, while the fluorescence emission area remained narrow.

In conclusion, here we demonstrate the application of ICG-HSA-loaded alginate hydrogel as a new surgical marker for noninvasive and real-time identification of tumor sites. In vivo animal studies in mouse and porcine models revealed promising results of this hydrogel system for prevision surgery of gastrointestinal tumors. This hydrogel system is expected to mark lesions using various contrast agents and serve as a drug delivery system following incorporation of suitable therapeutics.

\section{Acknowledgments}

Funding: This work was supported by the Ministry of Oceans and Fisheries (the project title: Development of marine material based near infrared fluorophore complex and diagnostic imaging instruments) and also by the National Cancer Center (1910070), Republic of Korea.

\section{Footnote}

Conflicts of Interest: The authors have no conflicts of interest to declare.

Open Access Statement: This is an Open Access article distributed in accordance with the Creative Commons Attribution-NonCommercial-NoDerivs 4.0 International License (CC BY-NC-ND 4.0), which permits the noncommercial replication and distribution of the article with the strict proviso that no changes or edits are made and the original work is properly cited (including links to both the formal publication through the relevant DOI and the license). See: https://creativecommons.org/licenses/by-nc-nd/4.0/.

\section{References}

1. Lu L, Zhou D, Jian X, Deng J, Yang P, Ding W. Laparoscopic colorectomy for colorectal cancer: retrospective analysis of 889 patients in a single center. Tohoku J Exp Med 2012;227:171-7.

2. Koh YX, Goh BKP. Minimally invasive surgery for gastric gastrointestinal stromal tumors. Transl Gastroenterol Hepatol 2017;2:108.

3. Aboosy N, Mulder CJ, Berends FJ, Meijer JW, Sorge AA. Endoscopic tattoo of the colon might be standardized to locate tumors intraoperatively. Rom J Gastroenterol 
2005;14:245-8.

4. Rogers BH. Colonoscopy with fluoroscopy. Gastrointest Endosc 1990;36:71-2.

5. Choi Y, Kim KG, Kim JK, Nam KW, Kim HH, Sohn DK. A novel endoscopic fluorescent clip for the localization of gastrointestinal tumors. Surg Endosc 2011;25:2372-7.

6. Tabibian N, Michaletz PA, Schwartz JT, Heiser MC, Dixon WB, Smith JL, Graham DY. Use of an endoscopically placed clip can avoid diagnostic errors in colonoscopy. Gastrointest Endosc 1988;34:262-4.

7. Ohdaira T, Konishi F, Nagai H, Kashiwagi H, Shito K, Togashi K, Kanazawa K. Intraoperative localization of colorectal tumors in the early stages using a marking clip detector system. Dis Colon Rectum 1999;42:1353-5.

8. Fu KI, Fujii T, Kato S, Sano Y, Koba I, Mera K, Saito H, Yoshino T, Sugito M, Yoshida S. A new endoscopic tattooing technique for identifying the location of colonic lesions during laparoscopic surgery: a comparison with the conventional technique. Endoscopy 2001;33:687-91.

9. Cohen JL, Forde KA. Intraoperative colonoscopy. Ann Surg 1988;207:231-3.

10. Frager DH, Frager JD, Wolf EL, Beneventano TC. Problems in the colonoscopic localization of tumors: continued value of the barium enema. Gastrointest Radiol 1987;12:343-6.

11. Coman E, Brandt LJ, Brenner S, Frank M, Sablay B, Bennett B. Fat necrosis and inflammatory pseudotumor due to endoscopic tattooing of the colon with india ink. Gastrointest Endosc 1991;37:65-8.

12. Park SI, Genta RS, Romeo DP, Weesner RE. Colonic abscess and focal peritonitis secondary to india ink tattooing of the colon. Gastrointest Endosc 1991;37:68-71.

13. Weissleder R, Ntziachristos V. Shedding light onto live molecular targets. Nat Med 2003;9:123-8.

14. Müller MG, Georgakoudi I, Zhang Q, Wu J, Feld MS. Intrinsic fluorescence spectroscopy in turbid media: disentangling effects of scattering and absorption. Appl Opt 2001;40:4633-46.

15. Sheng Z, Hu D, Zheng M, Zhao P, Liu H, Gao D, Gong P, Gao G, Zhang P, Ma Y, Cai L. Smart human serum albuminindocyanine green nanoparticles generated by programmed assembly for dual-modal imaging-guided cancer synergistic phototherapy. ACS Nano 2014;8:12310-22.

16. Ntziachristos V, Ripoll J, Wang LV, Weissleder R. Looking and listening to light: the evolution of whole-body photonic imaging. Nat Biotechnol 2005;23:313-20.

17. Saxena V, Sadoqi M, Shao J. Degradation kinetics of indocyanine green in aqueous solution. J Pharm Sci 2003;92:2090-7.

18. Deepthi S, Jayakumar R. Alginate nanobeads interspersed fibrin network as in situ forming hydrogel for soft tissue engineering. Bioact Mater 2017;3:194-200.

19. Andersen T, Auk-Emblem P, Dornish M. 3D Cell Culture in Alginate Hydrogels. Microarrays (Basel) 2015;4:133-61.

20. Somo SI, Langert K, Yang CY, Vaicik MK, Ibarra V, Appel AA, Akar B, Cheng MH, Brey EM. Synthesis and evaluation of dual crosslinked alginate microbeads. Acta Biomater 2018;65:53-65.

21. Lee KY, Mooney DJ. Alginate: properties and biomedical applications. Prog Polym Sci 2012;37:106-126.

22. Bidarra SJ, Barrias CC, Granja PL. Injectable alginate hydrogels for cell delivery in tissue engineering. Acta Biomater 2014;10:1646-62.

23. Liberale G, Vankerckhove S, Caldon MG, Ahmed B, Moreau M, Nakadi IE, Larsimont D, Donckier V, Bourgeois P; Group R\&D for the Clinical Application of Fluorescence Imaging of the Jules Bordet's Institute.. Fluorescence Imaging After Indocyanine Green Injection for Detection of Peritoneal Metastases in Patients Undergoing Cytoreductive Surgery for Peritoneal Carcinomatosis From Colorectal Cancer: A Pilot Study. Ann Surg 2016;264:1110-1115.

24. Shinde UP, Moon HJ, Ko du Y, Jung BK, Jeong B. Control of rhGH Release Profile from PEG-PAF Thermogel. Biomacromolecules 2015;16:1461-9.

25. Klohs J, Steinbrink J, Nierhaus T, Bourayou R, Lindauer U, Bahmani P, Dirnagl U, Wunder A. Noninvasive nearinfrared imaging of fluorochromes within the brain of live mice: an in vivo phantom study. Mol Imaging 2006;5:180-7.

26. Hyun JH, Kim SK, Kim KG, Kim HR, Lee HM, Park S, Kim SC, Choi Y, Sohn DK. A novel endoscopic fluorescent band ligation method for tumor localization. Surg Endosc 2016;30:4659-63.

Cite this article as: Lee SS, Kim H, Sohn DK, Eom JB, Seo YS, Yoon HM, Choi Y. Indocyanine green-loaded injectable alginate hydrogel as a marker for precision cancer surgery. Quant Imaging Med Surg 2020;10(3):779-788. doi: 10.21037/ qims.2020.02.24 